\title{
A!
}

This is an electronic reprint of the original article.

This reprint may differ from the original in pagination and typographic detail.

Badihi, Behnam; Liljemark, Aleksi; Sheikh, Muhammad Usman; Lietzen, Jari; Jantti, Riku

\section{Link Budget Validation for Backscatter-Radio System in Sub-1GHz}

Published in:

2019 IEEE Wireless Communications and Networking Conference, WCNC 2019

DOI:

10.1109/WCNC.2019.8885700

Published: 01/04/2019

Document Version

Peer reviewed version

Please cite the original version:

Badihi, B., Liljemark, A., Sheikh, M. U., Lietzen, J., \& Jantti, R. (2019). Link Budget Validation for Backscatter-

Radio System in Sub-1GHz. In 2019 IEEE Wireless Communications and Networking Conference, WCNC 2019 [8885700] (IEEE Wireless Communications and Networking Conference; Vol. 2019-April). IEEE.

https://doi.org/10.1109/WCNC.2019.8885700

This material is protected by copyright and other intellectual property rights, and duplication or sale of all or part of any of the repository collections is not permitted, except that material may be duplicated by you for your research use or educational purposes in electronic or print form. You must obtain permission for any other use. Electronic or print copies may not be offered, whether for sale or otherwise to anyone who is not an authorised user. 


\title{
Link Budget Validation for Backscatter-Radio System in Sub-1GHz
}

\author{
Behnam Badihi, Aleksi Liljemark, Muhammad Usman Sheikh, Jari Lietzén, Riku Jäntti \\ Department of Communications and Networking \\ Aalto University, Espoo, 02150 Finland. \\ Email:\{firstname.lastname\}@aalto.fi
}

\begin{abstract}
Ambient backscatter communications system (ABCS) has recently been introduced as a cutting edge technology in which devices communicate in wireless mode by exploiting ambient radio frequency (RF) signals instead of actively generating them. ABCS is a promising technology for Internet of Things (IoT) use cases in which the power efficiency is a major challenge yet to be addressed. ABCS is in its early development stages from theoretical and practical perspectives. In this regard, it is highly important to understand the multi-aspects of the link budget of ABCS. Hence, in this paper we conducted a comprehensive study including measurements in different propagation environment and thorough simulation. The measurements are preformed in sub-1 GHz band and particularly in $590 \mathrm{MHz}$ with the tags designed in the house. The results confirm the match between measurements and the simulations with trivial error.
\end{abstract}

Index Terms-Ambient backscatter communication system, link budget, IoT, bistatic dislocated backscatter, bistatic collocated backscatter, monostatic backscatter.

\section{INTRODUCTION}

The last several decades of revolutionary advances in embedded intelligence, connectivity, Internet related technologies and applications have brought pervasive computing devices. These devices with capability of sensing, actuating, monitoring and exchanging data are enormously increasing. Thanks to the recent technologies, the size of the smart objects, which are often mobile, are getting even smaller [1]. These objects, which are gradually becoming essential part of our life, communicate to each others and via Internet without human intervention and create novel applications and services. The ecosystem created by these objects is called Internet of Things $(I o T)$. By emerging IoT, the world is encountering a new paradigm shift in which the connectivity for human and houses will extend to the surrounding objects [2]. The final goal of such a system is to transform the Internet to a pervasive and ubiquitous one which at the end improves the quality of lives [3].

To make the IoT system fully functional and sustainable, yet many challenges and problems should be addressed. Among those, power efficiency is one of the most important challenges for the IoT as it defines the success and sustainability of the system. It is a very hard task to orchestrate such a network comprising of heterogeneous devices in a self-sustaining way without taking energy consumption into account . Furthermore, Energy is always an extra burden on the cost of the IoT devices and leaves environmental footprint. Therefore, designing a system with minimum power consumption or using energy efficient or battery-less devices are favorable. In this regard, backscatter communication system is considered as a potential solution for the energy challenge in IoT. This topic has recently gained quite much interest from academia and it can be considered as a leverage for green IoT applications in the near future [4].

The history of backscatter communication system goes back to 1948 when it was introduced by Harry Stockman [5]. Ever since it has continuously been studied and utilized in low-power low range wireless communication systems, for instance, in radio frequency identification (RFID) technology. The key idea in backscatter communication system is modulating the received RF carrier from the reader and reflecting it back to the reader instead of generating signal itself [6], [7]. However, due to limitations in this system, it can not extensively be used in the regular wireless communication systems for several reasons [8]. First of all, the communication is passive as the backscatter transmitter or tag always waits for the signal from the RF source to be triggered. Secondly, in the conventional backscatter system, the tags should be located close enough to the dedicated RF source, in particular, when passive tags are utilized, thus it limits the coverage area and device usage. At last but not least, since the most commercial readers in the conventional backscatter systems follow the monostatic architecture, they suffer from self-interference as the transmitter and receiver are collocated. Hence, solving the self-interference problem requires sophisticated design of the system, and in turn, it increases the cost of the system. These are some of the factors limiting the applicability of this system for a wide range of use cases for the IoT [9].

In order to overcome the above mentioned challenges, new methods have been suggested, namely, ambient backscatter communication system (AMBCS) [10] and bistatic backscatter communication system (BBCS) [11], [7], [12]. In AMBCS, the backscatter transmitter (or tag) modulates and backscatters the existing ambient RF signal such as digital television (DTV), FM broadcasting, or cellular base station signals. In BBCS, there is a dedicated carrier transmitter unlike AMBCS. Therefore, in BBCS, a large geographical area can be covered by using more than one carrier transmitter. In addition, based on [11], transmission power of carrier emitters can be reduced up to 50 times.

AMBCS solves the challenges related to conventional 


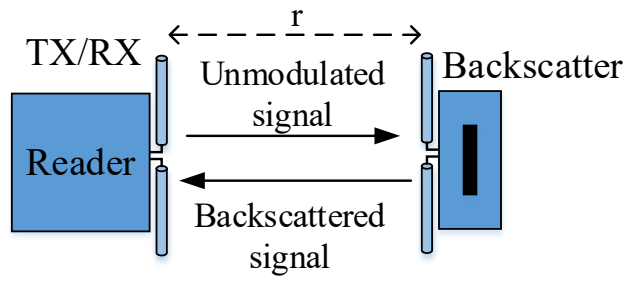

(a)

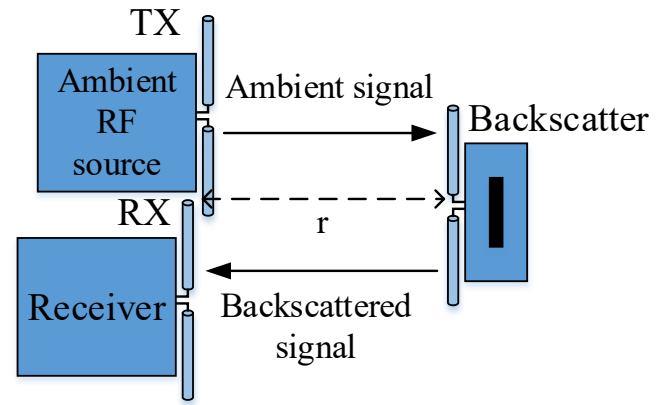

(b)

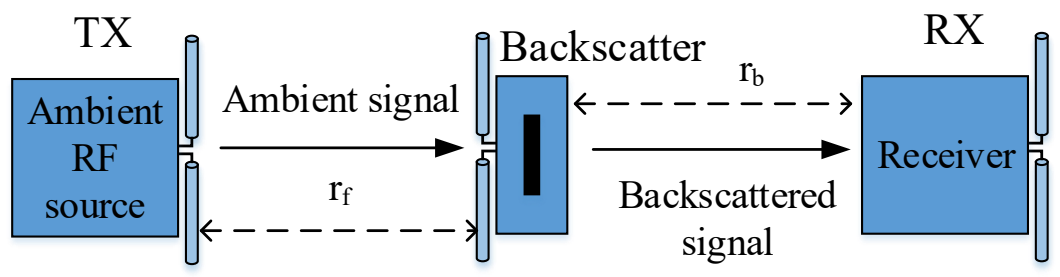

(c)

Fig. 1. Three main configurations of backscatter communication system: a) The monostatic backscatter, b) bistatic collocated backscatter, and c) bistatic dislocated backscatter configuration.

backscatter system in several ways. Firstly, the AMBCS uses an already existing signal in the atmosphere unlike the traditional backscatter system that uses RF signal broadcasted from the dedicated infrastructure. Secondly, AMBCS is an active system in which device to device communication occurs, while, in the traditional system, tag or backscatter should solely reply to the dedicated RF reader and only triggered when the reader transmits the signal in the forward link, hence it being considered as a passive system. Thirdly, the AMBCS system is a green technology with a very limited environmental footprint as it eliminates the need for expensive energy supply [10]. Finally, using the AMBCS is shown to increase the spectral efficiency since it can exist with the wireless legacy systems with imperceptible interference [13], [14].

Despite the fact that AMBCS and BBCS have many advantages as low range low power communication technologies for IoT use cases, they are still infant technologies in the early stage of their maturity. AMBCS and BBCS yet encounter many challenges that requires to be addressed before they are accommodated for IoT. Unlike the traditional backscatter system, AMBCS relies on ambient RF sources and thus it should be customized for specific signals and frequency bands. Furthermore, since AMBCS reflects the signal of the licensed sources, it should not interfere with the legacy users. In this regard, to understand these all, the deep knowledge of the link budget of backscatter system is required. This motivated us to study the link budget of the backscatter communication system using dedicated carrier transmitter in different environments and validate it with extensive simulations.

Several studies have been carried out on the link budget for backscatter communication system. Author in [15] presents the results of the measurement of excess link loss of RF tag in the vicinity of different materials in $915 \mathrm{MHz}$ band. A model is presented to calculate the read range in multi-path environment in [16]. Multipath fading measurements for multiantenna backscatter RFID at $5.8 \mathrm{GHz}$ band is carried out in [17] and the same author has studied the complete link budget for backscatter system in [18]. While in this paper, we study the link budget validation of the bistatic backscatter communication system via extensive measurements and simulations in different propagation environments.

The remaining part of the paper is organized as follows. Sec. II details an overview of backscattering communications system and related link budget. Thereafter, Sec. III illustrates the measurement and simulation setup and relevant parameters. The results of the paper are presented in Sec. IV and finally, conclusions are drawn in Sec. V.

\section{OVERVIEW OF BACKSCATTERING}

Three types of backscatter communication systems are reviewed in this section which are considered in the literature. Figure 1 illustrates these three categories of backscatter communication system with their corresponding link budget forms. These configurations are monostatic, bistatic collocated and bistatic dislocated backscatter communication system.

\section{A. The monostatic backscatter communication system (MBCS}

In the monostatic backscatter communication system (MBCS), the transmitter and receiver share a single antenna or in other words, the antenna of the system is a full duplex one. As the name suggests, this architecture comprises of two major components: backscatter transmitter (or tag) and a reader as 


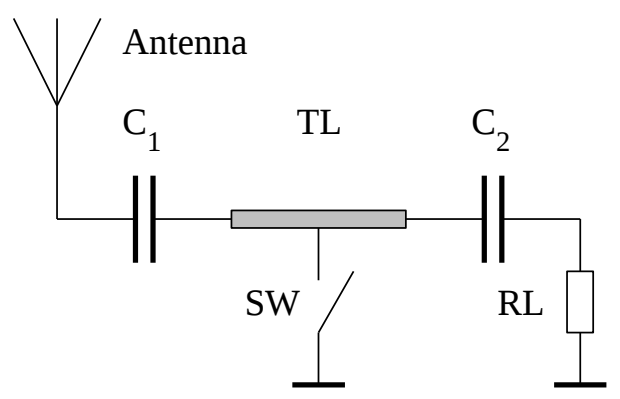

Fig. 2. Circuit diagram of the backscatter modulator.

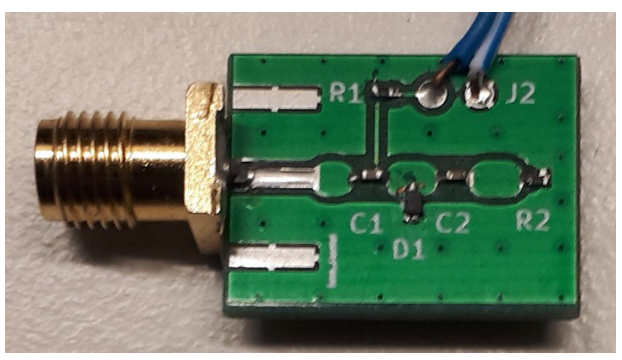

Fig. 3. Backscatter modulator.

shown in Fig. 1a. The backscatter device modulates the RF signal received from the reader and transmits the modulated signal back to the reader. As the forward link (i.e. from the RF source to the tag) and backscatter link (i.e. from tag to the reader) are identical paths, hence the received signal in the reader might suffer from severe path lost due to slow fading phenomena. According to [18], the received modulated backscatter power in the reader $P_{R}$ is given by a linear-scale link budget:

$$
P_{R}=\frac{P_{T} G_{T R}^{2} G_{B C}^{2} \lambda^{4} X^{2} M}{(4 \pi r)^{4} \Theta^{2} B^{2} F_{M B C S}},
$$

where $P_{T}$ is the power of unmodulated carrier transmitted form the reader, $G_{T R}$ and $G_{B C}$ are the load-matched freespace gain of the full-duplex antenna of the reader and the backscatter, respectively. Furthermore, $X$ stands for the polarization mismatch, $M$ denotes the modulation factor, $\Theta$ implies the on-object gain penalty of the backscatter transmitter, $B$ indicates the path-blockage loss, $F_{M B C S}$ symbolizes the monostatic backscatter paradigm and finally, $r$ shows the distance between the reader and backscatter.

The MBSC architecture is predominantly used in commercial RFID readers, while this configuration has a drawback of self-interference. Furthermore, monostatic configuration endures the round-trip path loss mentioned in [12] and doubly near-far problem meaning that if the backscatter located far from the reader, it undergoes higher outage probability due to signal loss between reader and backscatter[9].

\section{B. The bistatic collocated backscatter communication system (BCBCS)}

Unlike the MBSC, in bistatic collocated backscatter communication system (BCBCS), the RF power transmitter and receiver are separated as depicted in Fig. 1b. The antenna for transmitting and receiving are collocated within a few wavelength apart. Essentially, BCBCS can improve roundtrip path loss compared to MBSC as the forward link and backscatter link are slightly dissimilar. Also the design of this system is less complicated compared to monostatic one which leads to reduction in the cost.

The linear-scale link budget in BCBCS configuration for the received modulated backscatter power, $P_{R}$ is given by [18]:

$$
P_{R}=\frac{P_{T} G_{T} G_{R} G_{B C}^{2} \lambda^{4} X^{2} M}{(4 \pi r)^{4} \Theta^{2} B^{2} F_{B C B C S}},
$$

where $G_{T}$ and $G_{R}$ stand for the load-matched, free-space gain of transmitter and receiver antenna, respectively, and $F_{B C B C S}$ indicates the bistatic dislocated fade margin. Two major differences are observed by considering the Equation 1 and Equation 2. Firstly, different antenna gain for transmitter and receiver can exist as they are separated and secondly, since the small scale fading on forward path and backscatter path is different compared to monostatic, $F_{B C B C S}$ is applied in this configuration.

\section{The bistatic dislocated backscatter communication system (BDBCS)}

The final configuration of bacsctatter communication system is bistatic, dislocated backscatter in which receiver and transmitter antenna are freely located from the tag or backscatter device. This configuration is illustrated in Fig. 1c.

The linear-scale link budget in BDBCS for the received modulated backscatter power is calculated by:

$$
P_{R}=\frac{P_{T} G_{T} G_{R} G_{B C}^{2} \lambda^{4} X_{f} X_{B C} M}{(4 \pi)^{4} r_{f}^{2} r_{B C}^{2} \Theta^{2} B_{f} B_{B C} F_{B D B C S}},
$$

where $r_{f}$ and $r_{B C}$ are the forward path (from transmitter to the tag) and backscatter path (from tag to receiver), respectively, $X_{f}$ and $X_{B C}$ indicate forward link and backscatter link polarization mismatch, respectively, $B_{f}$ and $B_{B C}$ imply the forward link and backscatter link path-blockage loss, respectively, and finally, $F_{B D B C S}$ symbolizes the bistatic dislocated small-scale fading loss. Note that in the Equation 3, $G_{B C}$ is calculated as average RF tag gain. The reason being, the angle-of-arrival and angle-of-departure are dissimilar for wave incident entering to the tag and leaving from the tag [18].

\section{The backscatter modulator}

The backscatter modulator uses on-off keying (OOK) modulation. In OOK, the carrier frequency is switched on and off according to the binary representation of the message being transmitted. In this case, the received ambient signal is either reflected back or not, thus forming the OOK modulation. In order for the OOK modulation to work most efficiently, the 


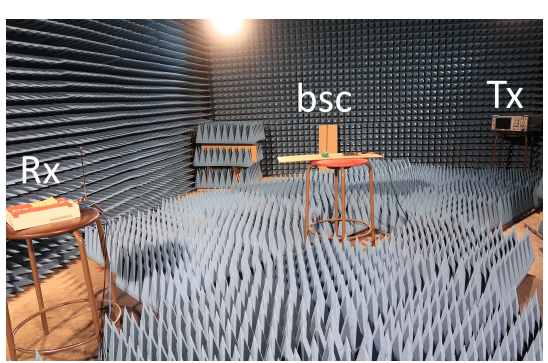

(a)

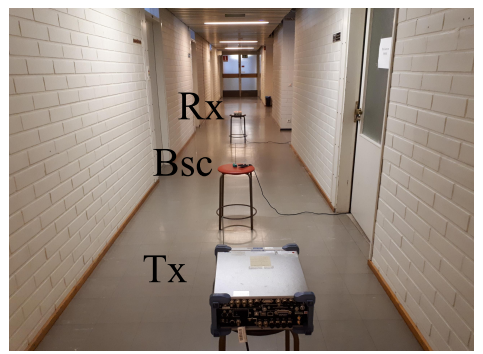

(b)

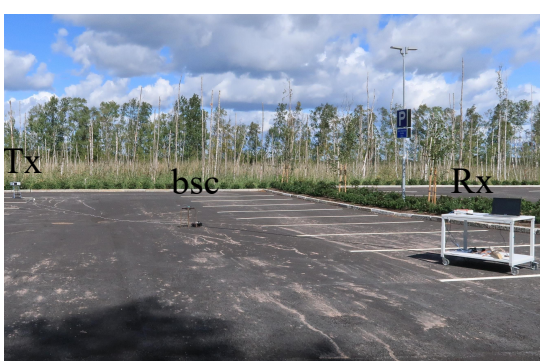

(c)

Fig. 4. The measurement setup in (a) anechoic chamber ; (b) corridor ; (c)outdoor.

difference between reflecting and non-reflecting states must be as high as possible. The circuit diagram of the backscatter modulator is in Fig. 2 and the modulator itself in Fig. 3. The modulator is a short length of $50 \Omega$ transmission line (TL), which is terminated with a $50 \Omega$ resistor (RL). This corresponds to the non-reflecting state. The reflecting state is realized using a current controlled switch (SW) to short-circuit the transmission line to the ground. The capacitors $\mathrm{C}_{1}$ and $\mathrm{C}_{2}$ are blocking $\mathrm{DC}$ voltage from entering either to the antenna or the terminating resistor.

For this experiment, the current controlled switch is realized by using a diode. The drawback of using a diode as a switching element is that it requires a constant current running through it to keep it conducting. The current in this case is in order of $10 \mathrm{~mA}$. Traditionally, a switching diode is biased backwards when it is not switched on. This is achieved by applying a negative voltage to it, thus minimizing the offstate capacitance. In this test setup only $10 \mathrm{~mA}$ control current drawn from a $+5 \mathrm{~V}$ output pin of a microprocessor is applied to the diode. This design of the backscatter modulator does not set limitations to the operating frequency. The limiting factor is the quality of the components used to manufacture the modulator.

With ideal components, the termination resistor is perfectly matched to the transmission line. The reflection coefficient in non-reflecting state is

$$
\rho=\frac{R L-Z_{0}}{R L+Z_{0}}=\frac{50 \Omega-50 \Omega}{50 \Omega+50 \Omega}=0
$$

Likewise with an ideal switch all power is reflected back. The switch's resistance $R_{\mathrm{SW}}$ when it is switched on is $0 \Omega$.

$$
\rho=\frac{R_{\mathrm{SW}}-Z_{0}}{R_{\mathrm{SW}}+Z_{0}}=\frac{0 \Omega-50 \Omega}{0 \Omega+50 \Omega}=-1
$$

The switching diode used in this experiment has a parasitic capacitance of $0.28 \mathrm{pF}$ when it is switched off and $0.6 \Omega$ forward resistance when it is switched on. The parasitic capacitance corresponds to a $963 \Omega$ capacitive reactance at $590 \mathrm{MHz}$. This reactance is in parallel with the $50 \Omega$ termination resistor and gives a return loss of $31.7 \mathrm{~dB}$ in the non-reflecting state. Correspondingly, the return loss in the reflecting state is determined by the forward resistance of the diode. The return loss in the reflecting state is $0.21 \mathrm{~dB}$. Other components and their imperfections have a smaller effect on the performance of the backscatter modulator than the switching diode.

\section{MEASUREMENT AND Simulation SETUP}

In our backscatter communication measurement setup, a single omni-directional transmit antenna with $0 \mathrm{dbi}$ antenna gain is connected to a SMBV100A Rohde \& Schwarz signal generator. The transmit power is set to $10 \mathrm{dBm}$, and the frequency of operation is $590 \mathrm{MHz}$. An RFID backscatter tag has an antenna with $0 \mathrm{dBi}$ antenna gain, and the RFID tag modulates the incoming signal from the transmitter to the receiver (reader). The transmission coefficient of the backscatter tag changes during the modulation (state A) and non-modulation (state B) period. The transmission coefficient of the backscatter tag is $-0.3 \mathrm{~dB}$ and $-26.87 \mathrm{~dB}$ during the state $\mathrm{A}$ and state $\mathrm{B}$, respectively. At the receiver end we have a software defined radio USRP-2932 connected to a single antenna with a $0 \mathrm{dBi}$ antenna gain. The SDR is in turn connected to a laptop for the post processing of the received signal. The height of the transmitter is $1.06 \mathrm{~m}$, and the height of the backscatter tag and the receiver antenna is $1 \mathrm{~m}$.

In this paper, we also would like to simulate and compare the simulation results with the measured results. Therefore, we model the simulation environment with as realistic parameters as possible, and use the same parameter values in simulations as used in our measurement setup. The values of transmission coefficient during state $\mathrm{A}$ and state $\mathrm{B}$ give a modulation factor of $-6.63 \mathrm{~dB}$ (0.217 linear value). For an indoor environment i.e. anechoic chamber and office corridor, fade margin of 5.56 $\mathrm{dB}$ and $4.77 \mathrm{~dB}$ are included in simulation for collocated and dislocated backscatter configuration, respectively. Whereas, a slightly higher fade margin of $7 \mathrm{~dB}$ is used in case of outdoor simulations. The transmit antenna is vertically polarized, therefore it is assumed that there is an average polarization mismatch loss of 0.65 between the transmitter and the tag, and between the tag and the receiver. Gain penalty and pathloss blockage loss are neglected and considered $0 \mathrm{~dB}$ loss for them.

The measurements are performed in three different environments, 1) Anechoic chamber, 2) Office corridor and, 3) Outdoor in a parking lot. In anechoic chamber, measurements are performed with bistatic collocated and bistatic dislocated configuration. It is important to mention that same set of equipment is used in all considered cases of measurements. 
In bistatic collocated configuration, both the transmitter and the receiver antennas were placed close to each other with a separation of $0.5 \mathrm{~m}$ only, and the received signal was measured at the receiver for the different position of the backscatter tag. As the size of the anechoic chamber is not large, therefore the distance between the transmitter and the RFID backscatter tag was limited to only 4 meters. Whereas, in bistatic dislocated backscatter configuration, the distance between the transmitter and the receiver was fixed at $4 \mathrm{~m}$, and the backscatter tag was moved between the transmitter and receiver in a straight line as shown in Fig. 4.

We have performed only bistatic dislocated configuration measurement in the corridor and outdoor environment. During the corridor and outdoor measurement, the separation between the transmitter and receiver was fixed to $8 \mathrm{~m}$ and $30 \mathrm{~m}$, respectively, and the position of the backscatter tag was changed during the measurement.

\section{RESULTS AND DISCUSSION}

In this section, a measured received backscatter signal power in anechoic chamber, office corridor and outdoor environment is provided, and a Root Mean Square (RMS) error between the predicted and the measured results is provided. Fig. 5 shows the received backscatter signal power for bistatic collocated and bistatic disclocated backscatter configuration as a function of distance between the transmitter and the backscatter device. In anechoic chamber, static measurements were done at the granularity of $0.25 \mathrm{~m}$ distance. In case of bistatic collocated configuration, it can be seen that as the backscatter tag moves away from the transmitter/reader the received backscatter power continue to attenuate as the transmitter and receiver are collocated. In the course of $4 \mathrm{~m}$ measurement path, the simulated results are optimistic as compared with the measured values during the first $2 \mathrm{~m}$ of the path, and then the simulated results becomes more pessimistic in the later part of the measurement route. However, in case of bistatic dislocated configuration the simulated results are in a good relationship with the measured values during the whole measurement route. The Root Mean Square (RMS) error between the measured and simulated values in the anechoic chamber are $3.48 \mathrm{~dB}$, and $1.5 \mathrm{~dB}$ for collocated and dislocated configuration, respectively.

In corridor measurement, the transmitter and receiver are separated by $8 \mathrm{~m}$ distance. In corridor environment there are other nearby reflecting walls, smooth reflecting ground surface, wooden doors, and glass doors. Therefore, due to multipath richness of environment the measured values exhibit more fading phenomenon. However, our simulated results with same parameter settings as selected for anechoic chamber measurement are in a close relationship with measured results. The RMS error between the measured and simulated values in the office corridor is $2.7 \mathrm{~dB}$ which is a bit higher as compared with anechoic chamber results.

In an outdoor parking lot, there is an open space with nearby trees and lamp posts. The transmitter and receiver are separated by $30 \mathrm{~m}$ distance. The static measurements with a

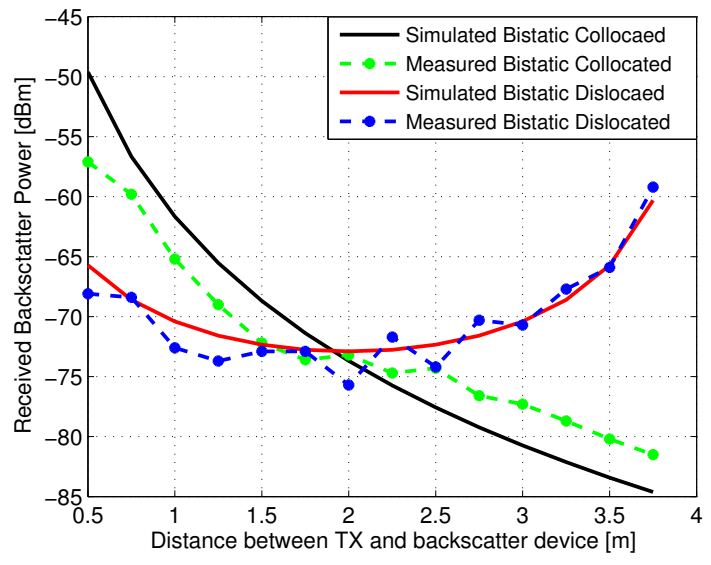

Fig. 5. Measured and simulated backscatter power in anechoic chamber.

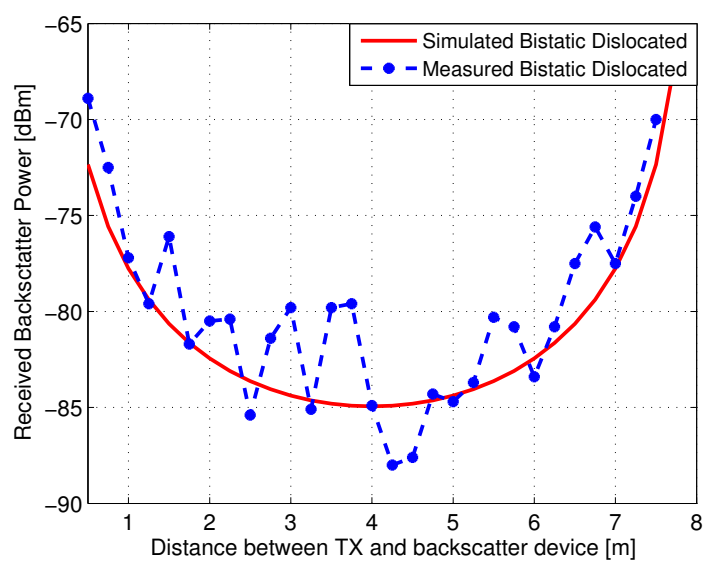

Fig. 6. Measured and simulated backscatter power in office corridor.

granularity of $1 \mathrm{~m}$ distance were performed. Due to the random nature of an outdoor environment, comparatively deeper fades are witnessed in an outdoor environment compared with an indoor environment, and this is why a higher value of fading margin is used in simulation for outdoor environment. The RMS error between the measured and simulated values in the outdoor environment is $2.93 \mathrm{~dB}$.

In the light of the measurement and simulated results for backscatter received power shown for three different environments in this paper, it can be said that if the link budget equations presented in this paper are used with realistic parameter values then they can provide a quite accurate and realistic result with small error of about $1.5 \mathrm{~dB}-2.93 \mathrm{~dB}$.

\section{CONCLUSION}

Backscatter communications system is an attractive technology in heterogeneous, large scale and self-sustainable networks. As such, it is very appealing for wireless sensor networks and Internet of Things (IoT) use cases. Backscatter communication system is in early stage of development and to make it fully functional many challenges related to this 


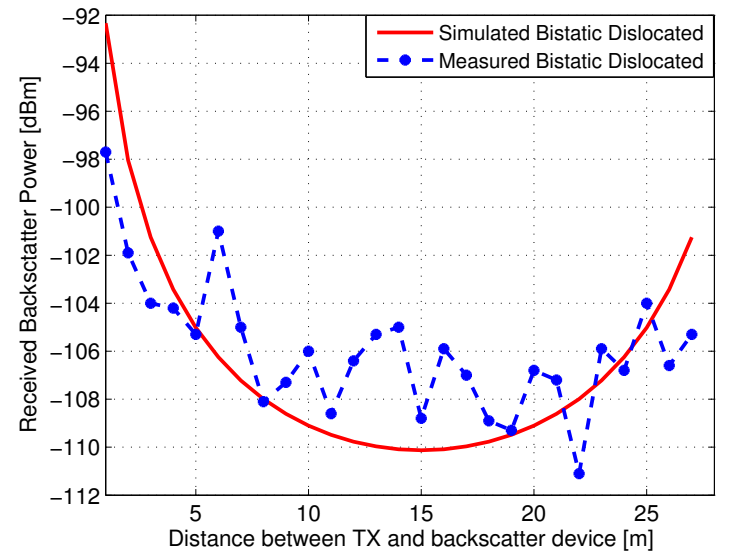

Fig. 7. Measured and simulated backscatter power in outdoor environment.

technology should be addressed. Hence, we comprehensively studied the link budget of bistatic backscatter communication system in different configurations in $590 \mathrm{MHz}$ band. We extensively performed simulations and measurements in different propagation environments. The results confirm the link budget equations in backscatter system with trivial error between measurements and simulations.

\section{REFERENCES}

[1] S. Gollakota, M. S. Reynolds, J. R. Smith, and D. J. Wetherall, "The emergence of rf-powered computing," Computer, vol. 47, no. 1, pp. 3239, Jan 2014.

[2] Y.-K. Chen, "Challenges and opportunities of internet of things," in Design Automation Conference (ASP-DAC), 2012 17th Asia and South Pacific. Citeseer, 2012, pp. 383-388.

[3] P. Kamalinejad, C. Mahapatra, Z. Sheng, S. Mirabbasi, V. C. Leung, and Y. L. Guan, "Wireless energy harvesting for the internet of things," IEEE Communications Magazine, vol. 53, no. 6, pp. 102-108, 2015.

[4] J. S. Ho, A. J. Yeh, E. Neofytou, S. Kim, Y. Tanabe, B. Patlolla, R. E. Beygui, and A. S. Poon, "Wireless power transfer to deep-tissue microimplants," Proceedings of the National Academy of Sciences, vol. 111, no. 22, pp. 7974-7979, 2014.

[5] H. Stockman, "Communication by means of reflected power," Proceedings of the IRE, vol. 36, no. 10, pp. 1196-1204, Oct 1948.

[6] A. Bletsas, S. Siachalou, and J. N. Sahalos, "Anti-collision backscatter sensor networks," IEEE Transactions on Wireless Communications, vol. 8 , no. 10,2009

[7] J. Kimionis, A. Bletsas, and J. N. Sahalos, "Bistatic backscatter radio for power-limited sensor networks," in 2013 IEEE Global Communications Conference (GLOBECOM), Dec 2013, pp. 353-358.

[8] P. Zhang, J. Gummeson, and D. Ganesan, "Blink: A high throughput link layer for backscatter communication," in Proceedings of the 10th international conference on Mobile systems, applications, and services. ACM, 2012, pp. 99-112.

[9] N. Van Huynh, D. T. Hoang, X. Lu, D. Niyato, P. Wang, and D. I. Kim, "Ambient backscatter communications: A contemporary survey," IEEE Communications Surveys \& Tutorials, 2018.

[10] V. Liu, A. Parks, V. Talla, S. Gollakota, D. Wetherall, and J. R. Smith, "Ambient backscatter: wireless communication out of thin air," in $A C M$ SIGCOMM Computer Communication Review, vol. 43, no. 4. ACM, 2013, pp. 39-50.

[11] J. Kimionis, A. Bletsas, and J. N. Sahalos, "Bistatic backscatter radio for tag read-range extension," in 2012 IEEE International Conference on RFID-Technologies and Applications (RFID-TA), Nov 2012, pp. 356361.

[12] J. Kimionis,A. Bletsas, and J. N. Sahalos, "Increased range bistatic scatter radio," IEEE Transactions on Communications, vol. 62, no. 3 , pp. 1091-1104, 2014.
[13] R. Duan, R. Jäntti, H. Yiğitler, and K. Ruttik, "On the achievable rate of bistatic modulated rescatter systems," IEEE Transactions on Vehicular Technology, vol. 66, no. 10, pp. 9609-9613, Oct 2017.

[14] D. Darsena, G. Gelli, and F. Verde, "Modeling and performance analysis of wireless networks with ambient backscatter devices," IEEE Transactions on Communications, vol. 65, no. 4, pp. 1797-1814, 2017.

[15] J. D. Griffin, G. D. Durgin, A. Haldi, and B. Kippelen, "Radio link budgets for $915 \mathrm{mhz}$ rfid antennas placed on various objects," in Texas Wireless Symposium, vol. 44, 2005.

[16] A. Lazaro, D. Girbau, and D. Salinas, "Radio link budgets for uhf rfid on multipath environments," IEEE Transactions on Antennas and Propagation, vol. 57, no. 4, pp. 1241-1251, 2009.

[17] J. D. Griffin and G. D. Durgin, "Multipath fading measurements for multi-antenna backscatter rfid at $5.8 \mathrm{ghz}$," in RFID, 2009 IEEE International Conference on. IEEE, 2009, pp. 322-329.

[18] J. D.Griffin, Joshua, G. D. Durgin, "Complete link budgets for backscatter-radio and rfid systems," IEEE Antennas and Propagation Magazine, vol. 51, no. 2, 2009. 